Section Editor

John J. Millichap, MD

Clinical Reasoning:

\title{
A 16-year-old girl with subacute weakness and sensory loss
}

\section{SECTION 1}

A previously healthy 16-year-old girl developed numbness and tingling in the lower extremities that progressed over a 10 -week period to the upper limbs. Two weeks prior to admission, she also developed weakness in her arms and legs, to the point of needing help getting dressed and support for walking. She denied sphincter or systemic symptoms. During the previous year, she had complained of intermittent shooting pains and tingling down her arms. Her psychiatric history was relevant for obsessive-compulsive traits and depression, and family history included autoimmune diseases in 2 sisters, an uncle, and 2 grandparents.

Upon examination, higher mental functions and cranial nerves were intact. The patient had upper limb predominant quadriparesis. Muscle strength was $4 / 5$ proximally and $3 / 5$ distally in upper limbs and $4 / 5$ throughout the lower limbs. Temperature and vibration sensations were mildly reduced in the upper limbs and moderately reduced in the lower limbs. Tone was increased in all limbs, and deep tendon reflexes (DTRs) were $3+$ in upper limbs and $4+$ in lower limbs. Hoffmann sign and extensor plantar responses were present bilaterally. Romberg sign was positive and the patient could not walk without support due to a combination of motor and sensory changes.

\section{Questions for consideration:}

1. Where would you localize the lesion based on her symptoms and neurologic examination?

2. What would be your differential diagnosis and what workup would you order? 


\section{SECTION 2}

Multimodal sensory changes and weakness involving lower and upper limbs, with increased tone, hyperactive DTRs, and plantar extensor responses, suggest an upper motor neuron lesion. Further, the lesion can be suspected to be at the proximal cervical cord level due to tetraparesis with intact cranial nerves and cognitive functions. Cervical lesions can present with symptoms restricted to the lower limbs due to the topographic distribution of fibers from lower spinal segments. Shooting pain is a classical feature in spondylotic myelopathy, but may also be present in other etiologies. Considering symptoms had been progressing over 10 weeks, the patient's diagnosis was consistent with a subacute progressive cervical myelopathy.

With acute presentation, emergent spinal MRI should be performed to exclude compressive causes that may require urgent surgery, such as epidural hematoma or disc herniation. The differential diagnosis of myelopathy is extensive and rapidity of symptoms will help to narrow it down. For acute transverse myelitis, criteria include demonstration of inflammation within the spinal cord
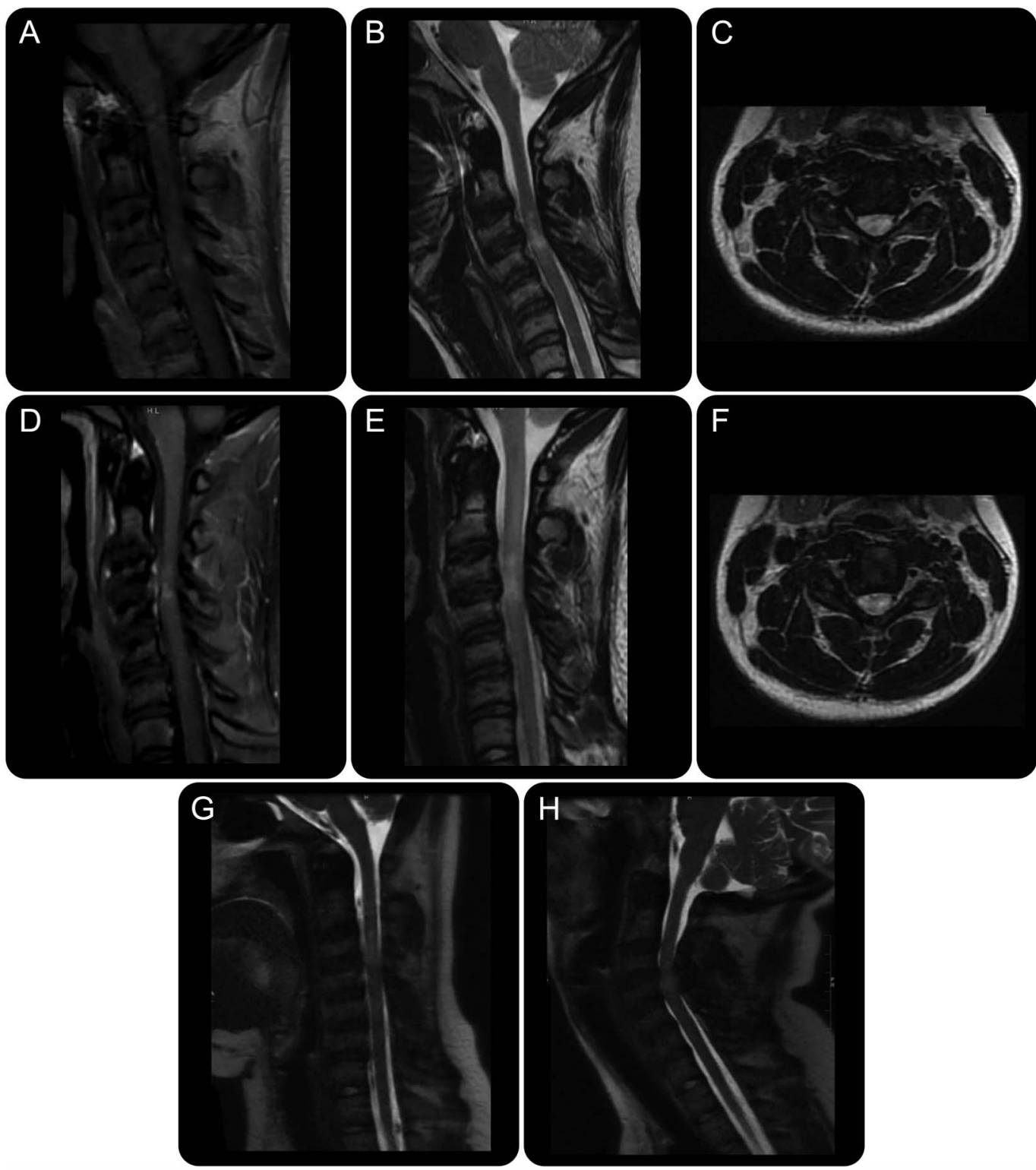

Sagittal T1-weighted imaging after contrast injection (A), sagittal T2 (B), and axial T2 MRI of cervical spine at presentation (C) show T2 signal hyperintensity in the cord that extends from C3 to C5 with multifocal intramedullary enhancement in C3C4, signs of disc desiccation, C3-C4 disc protrusion, and moderate canal stenosis. Similar sequences 5 months later (D-F) demonstrate increased enhancement. Dynamic MRI T2 sequence of the spine demonstrates progressive spinal canal narrowing at C3-4 with neck extension $(\mathrm{H})$ compared to neutral flexion $(\mathrm{G})$ position, related to combination of progressive disc bulge and ligamentum flavum buckling. 
(CSF pleocytosis, gadolinium enhancement, or elevated immunoglobulin $\mathrm{G}$ [IgG] index), exclusion of extra-axial compression, and time to peak of maximum disability greater than 4 hours and less than 21 days. ${ }^{1}$ Peak of symptoms less than 4 hours is uncommon and suggests a vascular cause, including fibrocartilaginous embolism. Acute myelopathies can be of inflammatory or infectious origin while more progressive courses should raise suspicion for toxic, metabolic, hereditary, or neoplastic causes. Tumors will usually present with a progressive course but can present acutely if an intratumoral hemorrhage or infarction occur. Similarly, arteriovenous malformations can present with fluctuating or progressive symptoms due to vascular steal or gradual ischemia.

MRI of the neuroaxis revealed a T2-bright cervical cord lesion at C3-C5 with inhomogeneous contrast enhancement and multilevel degenerative changes of the cervical spine that caused moderate cervical narrowing on standard MRI (figure, A-C). CSF analysis revealed clear liquid, total protein of $33 \mathrm{mg} / \mathrm{dL}$, glucose of $49 \mathrm{mg} / \mathrm{dL}, 1$ leukocyte (100\% lymphocyte), no oligoclonal bands, IgG index of 0.5 (in the normal range), no bacteria on Gram staining, and negative cultures. A rapid qualitative PCR for herpes simplex virus (HSV)-1 was positive but negative on confirmatory quantitative real-time PCR. PCR for enteroviruses and other herpesviruses were negative. Antinuclear antibodies (ANA) were positive at a $1 /$ 320 titer, but negative for ANA subtypes. C-reactive protein and erythrocyte sedimentation rate were normal. Serum AQP4-IgG, rheumatoid factor, antiphospholipid antibodies, and extensive infectious serologies were also negative. Serum vitamin $B_{12}$ was $299 \mathrm{pg} / \mathrm{mL}$; however, methylmalonic acid and homocysteine levels were normal. Serum copper level was normal. Based on radiologic features, our patient was presumed to have an inflammatory or infectious cause, although the chronology of symptoms was unusual for these etiologies.

\section{Question for consideration:}

1. What is the differential diagnosis of an enhancing cord lesion with such a clinical course? 


\section{SECTION 3}

Intramedullary signal change with enhancement can be found in neoplastic, paraneoplastic, vascular, inflammatory, infectious, and compressive etiologies. Brain imaging should be performed to exclude accompanying abnormalities seen in inflammatory disorders such as acute disseminated encephalomyelitis, multiple sclerosis, neuromyelitis optica spectrum disorders, systemic lupus erythematosus (SLE), Sjögren syndrome, Behçet disease, sarcoidosis, and some infectious etiologies. However, an isolated myelitis can occur as the presenting symptom in the absence of other CNS MRI abnormalities. Inflammatory diseases such SLE or Sjögren syndrome include long T2 hyperintense central cord lesion with expansion and variable enhancement, although sometimes may be multifocal. Arteriovenous malformations can present with intramedullary signal change and will often show abnormal flow voids on MRI. Some intramedullary tumors can present with a relative acute course mimicking transverse myelitis, and may be challenging to distinguish radiologically. In addition, response to glucocorticosteroid treatment can be seen in certain tumors, such as in intramedullary lymphoma, which are typically seen in older adults. Tumors will usually enlarge cord diameter while inflammatory lesions rarely do so. A nearby syrinx may develop when the tumor obstructs the central canal. In inflammatory myelopathies, maximum disability is usually reached before 3 weeks from onset, and CSF will frequently show increased leukocyte counts and intrathecal antibody synthesis (oligoclonal bands not present in serum, increased IgG index). Ongoing clinical worsening or contrast enhancement beyond this time frame should prompt searching for alternative diagnoses. Exceptions include spinal cord sarcoidosis, where normal CSF and persistence of enhancement may be seen. Typical radiologic features such pial involvement and systemic findings may help with the diagnosis. ${ }^{2}$ Hirayama disease is another cause of progressive distal-predominant upper limb weakness, albeit classically in young adults, and is restricted to upper limbs with asymmetric involvement and no sensory disturbances.
MRI of cervical spine would show focal spine atrophy and shifting of the posterior dural sac during cervical flexion.

The patient was diagnosed with transverse myelitis of unclear origin but based on radiologic features and initial positive PCR, HSV-1 was suspected. She had no other signs of infection. She was treated with IV acyclovir for 14 days; however, confirmatory HSV-1 PCR returned negative. She also received an overlapping course of high-dose IV methylprednisolone for 5 days. The patient did not have substantial improvement and was discharged home with physical therapy. Two months later, the patient developed further weakness and repeat cervical MRI showed increased size of the T2-bright lesion and increased enhancement at C3-C4 (figure, D-F). Repeat CSF analysis was unremarkable, with no oligoclonal bands and normal IgG index. CSF quantitative PCR for HSV-1 and serum HSV-1 IgG and immunoglobulin M were negative. Repeat serum AQP4-IgG was negative. However, in light of positive ANA, clinical worsening, ongoing enhancement, and substantial family history of autoimmune disease, an autoimmune etiology was still suspected and the patient underwent 4 sessions of plasma exchange without any clinical response.

At this point, the patient's history and MRI findings were revisited. The notable degenerative changes in discs and vertebrae were unexpected for her age. Upon further questioning, the patient denied acute cervical injury history but was found to have a motor tic for the previous 11 years described as "taking her right hand to the left side of her head; pulling in a downward and ipsilateral motion and then alternating sides." This tic occurred up to several times per hour by the time of presentation. Psychiatric evaluation revealed past and present history of other motor tics (i.e., cracking her knuckles), depressive symptoms, and obsessive-compulsive trait, but did not meet the criteria for obsessive-compulsive disorder or Tourette syndrome. Patient tics were managed with Comprehensive Behavioral Intervention for Tics.

\section{Question for consideration:}

1. What is the main pattern of MRI enhancement in patients with spondylotic myelopathy? 


\section{SECTION 4}

Spondylotic myelopathies can present with intramedullary T2 signal change that may extend longitudinally further from the site of compression, with an enhancing band below the level of maximum stenosis, often referred to as pancake-like. ${ }^{3}$ These findings may mimic transverse myelitis leading to erroneous treatment. ${ }^{4}$ Interestingly, enhancement may persist despite successful decompression and clinical recovery. ${ }^{2,5}$ Contrast enhancement reflects disruption of the blood-spinal cord barrier at that level, but the exact mechanisms are not wellunderstood. ${ }^{6}$ Cervical spondylotic changes are unusual in adolescents and children and should raise the issue of traumatic history. Further confusion may occur when spondylotic changes are interpreted to be too mild to cause myelopathy, since dynamic factors not seen on static imaging may be involved.

In the case of our patient, flexion and extension cervical spine radiographs did not show signs of instability; however, dynamic MRI of the neck unveiled prominent cervical canal narrowing during neck extension (figure, $\mathrm{G}$ and $\mathrm{H}$ ). The patient underwent C3-C5 laminoplasty, and over an 8-month follow-up, the neurologic deficits stabilized and the neuropathic pain notably improved.

Motor tics involving the neck as a cause of repetitive low-grade trauma leading to cervical spondylotic myelopathy have been scarcely reported; most of the descriptions are in young adults, and in the setting of Tourette syndrome. ${ }^{8}$ Other movement disorders reported to cause spondylotic myelopathy include cervical dystonia. ${ }^{9}$ However, our patient had no major psychiatric disturbances at the time of presentation, and the presence of a tic was not initially noted.

This case illustrates an unusual presentation of a traumatic myelopathy, in which MRI features and absence of acute trauma led to the diagnosis of suspected transverse myelitis and delayed surgical decompression. This case highlights the critical importance of careful history and bedside examination and observation to guide diagnosis.

\section{AUTHOR CONTRIBUTIONS}

Dr. Quintanilla-Bordás: drafting/revising the manuscript and design, acquisition of data, analysis and interpretation. Dr. Nourbakhsh: critical revision of the manuscript. Drs. Strober and Raffel: acquisition of data, analysis and editing of the manuscript. Dr. Waubant: study concept and design, critical revision of the manuscript for important intellectual content, supervision.

\section{STUDY FUNDING}

No targeted funding reported.

\section{DISCLOSURE}

The authors report no disclosures relevant to the manuscript. Go to Neurology.org for full disclosures.

\section{REFERENCES}

1. Transverse Myelitis Consortium Working Group. Proposed diagnostic criteria and nosology of acute transverse myelitis. Neurology 2002;59:499-505.

2. Flanagan EP, Krecke KN, Marsh RW, et al. Specific pattern of gadolinium enhancement in spondylotic myelopathy. Ann Neurol 2014;76:54-65.

3. Flanagan EP, Marsh RW, Weinshenker BG. Teaching NeuroImages: "Pancake-like" gadolinium enhancement suggests compressive myelopathy due to spondylosis. Neurology 2013;80:e229.

4. Kelley BJ, Erickson BJ, Weinshenker BG. Compressive myelopathy mimicking transverse myelitis. Neurologist 2010;16:120-122.

5. Cabraja M, Abbushi A, Costa-Blechschmidt C, et al. Atypical cervical spondylotic myelopathy mimicking intramedullary tumor. Spine 2008;33:e183-e187.

6. Ozawa H, Sato T, Hyodo H, et al. Clinical significance of intramedullary Gd-DTPA enhancement in cervical myelopathy. Spinal Cord 2010;48:415-422.

7. Zeitoun D, El Hajj F, Sariali E, et al. Evaluation of spinal cord compression and hyperintense intramedullary lesions on T2-weighted sequences in patients with cervical spondylotic myelopathy using flexion-extension MRI protocol. Spine J 2015;15:668-674.

8. Dobbs M, Berger JR. Cervical myelopathy secondary to violent tics of Tourette's syndrome. Neurology 2003;60: 1862-1863.

9. Fung GPG, Chan KY. Cervical myelopathy in an adolescent with Hallervorden-Spatz disease. Pediatr Neurol 2003; 29:337-340 


\section{Neurology}

\section{Clinical Reasoning: A 16-year-old girl with subacute weakness and sensory loss}

Carlos Quintanilla-Bordás, Bardia Nourbakhsh, Jonathan Strober, et al.

Neurology 2017;88;e225-e229

DOI 10.1212/WNL.0000000000004010

\section{This information is current as of June 5, 2017}

\section{Updated Information \& Services}

References

Subspecialty Collections

Permissions \& Licensing

Reprints including high resolution figures, can be found at: http://n.neurology.org/content/88/23/e225.full

This article cites 9 articles, 3 of which you can access for free at: http://n.neurology.org/content/88/23/e225.full\#ref-list-1

This article, along with others on similar topics, appears in the following collection(s):

Clinical neurology history

http://n.neurology.org/cgi/collection/clinical_neurology_history MRI

http://n.neurology.org/cgi/collection/mri

Spinal cord trauma; see Trauma/spinal cord trauma

http://n.neurology.org/cgi/collection/spinal_cord_trauma-see_trauma-s pinal_cord_trauma

Tics

http://n.neurology.org/cgi/collection/tics

Transverse myelitis

http://n.neurology.org/cgi/collection/transverse_myelitis

Information about reproducing this article in parts (figures,tables) or in its entirety can be found online at:

http://www.neurology.org/about/about_the_journal\#permissions

Information about ordering reprints can be found online:

http://n.neurology.org/subscribers/advertise

Neurology ${ }^{\circledR}$ is the official journal of the American Academy of Neurology. Published continuously since 1951 , it is now a weekly with 48 issues per year. Copyright @ 2017 American Academy of Neurology. All rights reserved. Print ISSN: 0028-3878. Online ISSN: 1526-632X.

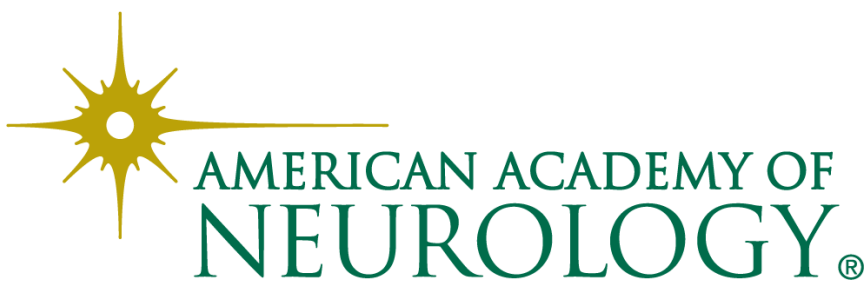

